\title{
GIW and InCoB are advancing bioinformatics in the Asia-Pacific
}

\author{
Christian Schönbach ${ }^{1,2^{*}}$, Paul Horton ${ }^{3,4}$, Siu-Ming Yiü ${ }^{5}$, Tin Wee Tan ${ }^{6}$, Shoba Ranganathan ${ }^{7,6}$ \\ From Joint 26th Genome Informatics Workshop and Asia Pacific Bioinformatics Network (APBioNet) 14th \\ International Conference on Bioinformatics (GIW/InCoB2015) \\ Tokyo, Japan. 9-11 September 2015
}

\begin{abstract}
GIW/InCoB2015 the joint 26th International Conference on Genome Informatics (GIW) and 14th International Conference on Bioinformatics (InCoB) held in Tokyo, September 9-11, 2015 was attended by over 200 delegates. Fifty-one out of 89 oral presentations were based on research articles accepted for publication in four BMC journal supplements and three other journals. Sixteen articles in this supplement and six articles in the BMC Systems Biology GIW/InCoB2015 Supplement are covered by this introduction. The topics range from genome informatics, protein structure informatics, image analysis to biological networks and biomarker discovery.
\end{abstract}

\section{Introduction}

The International Conference on Bioinformatics ( $\mathrm{InCoB}$ ) is annual conference of the Asia-Pacific Bioinformatics Network [1], while GIW is the annual conference of the Association of Asian Societies for Bioinformatics (AASBi) [2]. Both GIW and InCoB are intimately associated with the development, growth and maturation of bioinformatics in Asia. Twenty-six years after the first GIW and fourteen years after the first InCoB, bioinformatics is now a well-established and diverse discipline within life and computer sciences. Hence, the differences in thrust and focus areas of the two conferences (e.g. genome informatics for GIW) faded over time. In the past five years, GIW and InCoB had to cope with increasingly tight travel budgets of researchers who are subject to stringent key performance indicators often requiring their work presented in talks to be published as articles in journals with sufficiently high impact factors. As both conferences draw submissions from a largely overlapping clientele, APBioNet and AASBi member society Japanese Society for Bioinformatics (JSBi) decided to organize a joint GIW/InCoB2015 conference [3] rather than competing for submissions and potential delegates. The

\footnotetext{
* Correspondence: christian.schoenbach@nu.edu.kz

'Department of Biology, School of Science and Technology, Nazarbayev University, Astana 010000, Republic of Kazakhstan

Full list of author information is available at the end of the article
}

immediate effects of joining forces were the ability to offer manuscript submission to seven journal tracks (Bioinformatics [4], BMC Genomics [5], BMC Bioinformatics [6], BCM Medical Genomics [7], BMC Systems Biology [8], IEEE/ACM Transactions on Computational Biology and Bioinformatics [9] and Journal of Computational Biology and Bioinformatics [10]) and savings in logistics, operational and administrative costs that allowed us to sustain moderate registration fees.

As bioinformaticians we are familiar with network topologies. If we treat conference stakeholders similar to vertices and their connections in dynamic networks and try to optimize them we can achieve a state of scale-free conferences. APBioNet considered GIW/InCoB2015 a test run for a future, potentially larger, joint multi-partner supraregional bioinformatics conference in Asia. The expected economy of scale and richness in diversity of topics will be beneficial for all stakeholders in the conference: participants, invited speakers, organizers/hosts, publishers, sponsors, funders, exhibitors, venues, transport and accommodations.

\section{Manuscript submission and review}

All submitted manuscripts were reviewed by at least two reviewers before a decision on rejection, major revision or minor revision was reached. Details on the reviewing process, acceptance, the list of manuscripts selected for 
the Best Paper Awards and the names of the program committee members are available in the Introduction of the BMC Genomics GIW/InCoB2015 supplement [11]. An overview of the 16 articles in this supplement [6] and six articles in BMC Systems Biology supplement [8] is given in the next sections.

\section{Biomarkers and disease networks}

The wider application of biomarkers in diagnosing complex diseases and their progression is still limited by suboptimal accuracy and stability. Two studies addressing these issues apply network and algorithm approaches to cardioembolic stroke and Alzheimer's disease data. Wong et al. [12] constructed protein-protein interaction networks based on temporal gene expression profiles derived from microarray time course data of cardioembolic stroke. Pathway analyses combined with a stroke-relevant scoring function revealed network-based biomarkers that were common or distinct for three post-stroke time points. Vandewater et al. [13] tackled the problem of combinatorial solution space in finding robust blood-based biomarker and demographic feature combinations that are predictive for the progression of cognitive impairment towards Alzheimer's disease. An adaptive genetic algorithm with logistic regression approach resulted in a 30 feature model that showed superior performance in predicting progression to both mild cognitive impairment and Alzheimer's disease.

Another common challenge in biomarker discovery for complex diseases is that high-throughput transcriptome, proteome and metabolome data are amenable for subtyping (e.g. cancer) and sub-processes in the disease but require more than Gene Ontology-based functional enrichment to understand the underlying molecular mechanisms. Wu et al. [14] designed a large-scale textmining system for thyroid cancer sub-classification and detailed molecular pathway understanding which appears to be sufficiently generic to be applied to other complex diseases.

Copy number alterations (CNAs) are known to contribute to the deregulation of gene expression in cancers [15]. Piccetti et al. [16] developed a methodology related to learning cooperative regulation networks from gene expression data [17] to derive for each gene and sample of bladder cancer with CNAs a network-wide deregulation score. The approach is expected to provide useful information on shared and different deregulation types among cancer subtypes as well as individual patients. Taguchi [18] applied principal component analysis with unsupervised feature extraction to identify deregulated genes and aberrantly methylated promoters in the endocrine inhibitor vinclozolin exposed rat F3 cell lineages representing different developmental stages. His approach yielded candidate chemokine signaling pathways and several leucine-rich repeat proteins that may play a role in transgenerationalmediated epigenetic diseases.

\section{Transcriptional regulation and protein-protein interactions}

Multicellular human and unicellular yeast share core eukaryotic pathways that can be exploited in humanized yeast cell [19] disease models and study of transcriptional regulation. Wu et al. [20] implemented a onestop web-based yeast-associated genes mining tool YAGM for biological associations that include TF binding, regulation, mutant phenotype, physical and genetic interactions among others.

Functional redundancy of TFs in yeast has been studied experimentally using knock-out strains indicating that only a small percentage of binding sites regulated by a particular TF are affected when the TF is knocked-out. Wu et al. [21] reanalyzed the functional redundancy for molecular features that may explain why one TF can compensate for another one. Features that rendered a TF redundant included low expression levels, TF binding sites in close vicinity of transcription start sites, few bound TFs among other factors. Benchmarking of new or modified computational methods of TF binding predictions can become time consuming when done for all methods. Lai et al. [22] developed a cooperative TF pair evaluator that incorporated fourteen different methods and their performance indices.

Protein-protein interactions (PPIs) can change spatially and temporally depending on the context. When PPIs are clustered, the temporal and/or spatial differences that delineate molecular biological information can be lost. Stoney et al. [23] designed a clustering strategy that can accommodate context dependent PPIs utilizing Gene Ontology annotations or sequence homology to determine functional communalities. The approach allows PPIs to be represented in multiple pathways rather than one node with multiple connections, a prerequisite for exploring functional organization and changes.

The work of Konishi [24] also addresses clustering in the presence of a temporal dimension. The scaled principal component analysis of mammary gland development microarray time course data showed robustness to noise and separation of groups across all time points.

\section{Protein structures and post-translational modifications}

Post-translational modification (PTM) or the absence of it affects the structure of a protein. Potential PTM-mediated changes in protein-protein binding characteristics may alter the regulation of protein networks but also make PTM a drugable target in diseases pathways. For example, aberrant neddylation has been reported to be involved in several cancers, cardiac and neurodegenerative diseases [25]. 
Yavuz et al. [26] developed a new neddylation site prediction method using a support vector machine algorithm. The feature selection includes protein properties beyond sequence conservation such as hydrophobicity, disorder state and various physicochemical properties. The second PTM prediction method and tool reported in this supplement utilizes maximal dependence decomposition of potential motifs associated with O-linked glycosylation catalyzed by O-GlcNAc transferase [27].

Sowmya and Ranganathan [28] investigated generic features of PPIs that govern binding at the interface of proteins on a quantitative level. Among nine feature classes: interface area, interface polar abundance, interface charged residues percentage, and solvation free energy gain upon interface formation, binding energy turned out to be significantly different. The finding has the potential to improve the characterization of protein complexes at the single-residue level and improve docking methods for predicting protein-protein binding. Large-scale prediction of protein-binding affinities based on sequence properties was addressed by Srinivasulu et al. [29] who introduced an SVM with support vector regression feature selection. Fourteen features of physiochemical properties were found to be informative for predicting the affinities of heterodimeric protein complexes. The last paper in this section deals with the issue of antigenic protein surface residues in conformational B-cell epitope prediction. Ren et al. [30] developed a weighted SVM algorithm that has been implemented with together with data pre-processing steps as the positive-unlabeled prediction pipeline (PUPre). PUPre performance was evaluated using unbound antigen structures of antigens that circumvents constraints of bound antigen structures. PUPre outperformed existing methods when tested on the three antigens with known epitopes, and it will be interesting to see if the method will be implemented as web tool for use in prediction of unknown B-cell epitopes by a wider user community.

\section{Bioimaging}

Biological image classification is gaining more and more importance beyond cell imaging and diagnostics. Artificial neural networks (ANN) have been successfully applied in the taxonomic classification of algae from images [31]. Here, Kien et al. [32] developed an automated ANN-based species identification technique for copepod zooplankton using dorsal microscopy images. The methodology shows promise to reduce the time spent on the taxonomic analysis of plankton in aquatic ecosystem studies. The focus of the second bioimaging paper is on single-cell Raman spectra of bacteria [33]. The authors present an improved method that transforms the Raman spectrum into a discrete spectrum that results into rapid spectra comparison and higher classification accuracy.

\section{Genomics}

Light-RCV [34] is another next-generation sequencing reads and alignment viewer. The genome-wide read coverage at base level is facilitated by a memory dump of the coverage which does not require lengthy sequential loading of a specific base position. Kimura and Koike [35] used the Burrows-Wheeler transform to identify the exact position of break points in genomic rearrangements. The exact position is determined without using split reads by applying discordant pairs and a lossless dictionary of reads. When applied to heterogeneous cancer genome sequence samples previously unknown somatic breakpoints were detected. Genomics is increasingly routinely applied in environmental sciences. For example in soil microbiome studies $16 \mathrm{~S}$ rDNA sequencing is used to determine taxonomic distribution of bacteria in samples. Chen et al. [36] designed a sequencing pipeline with reduced primer bias and post-processing that resulted in longer 16S rDNA sequences during a proof-of-principle sequencing of dioxin-containing soil samples. In microbial metagenomic studies the number of mixed bacterial populations is termed richness. Jayasundara et al. [37] improved the estimation of richness for viral strains using quasispecies spectra obtained with a new probabilistic method.

\section{Privacy}

In 2012 the US Presidential Commission for the Study of Bioethical Issues released a report on "privacy and progress in whole genome sequencing" [38]. The analysis results and recommendations range from policies and ethics to technical issues such as computational access. Database developers and providers face the conundrum of promoting data access and sharing data while providing protection, security and privacy. In the past three years a few papers explored fuzzy encryption to deal with privacy issues when searching genotype and singlenucleotide polymorphism (SNP) data [39,40]. Shimizu et al. [41] proposed an additive-homomorphic cryptosystem for protecting user and database privacy. The results of a case-study on searching a large chemical compound database have been encouraging and may result in future applications in drug discovery-related searching of sensitive data and searching for SNPs in personal genome databases.

\section{Conclusion}

The articles in the two GIW/InCoB2015 supplements of BMC Bioinformatics and BMC Systems Biology represent a variety of new bioinformatics methods that will enable new studies to advance our biological and biomedical knowledge on molecular and system levels. The next opportunity to present and publish innovative bioinformatics tools and novel bioinformatics-driven 
research in the framework of $\mathrm{InCoB}$ and collaborating partners is coming soon. InCoB2016 will be held from September 21-23, 2016 in Singapore [42].

\section{List of abbreviations used}

AASBi - Association of Asian Societies for Bioinformatics; ANN - artificial neural network; APBioNet- Asia-Pacific Bioinformatics Network; CNA - copy number alteration; GIW - International Conference on Genome Informatics; HMM - Hidden Markov Model; IEEE/ACM IEEE Communications Society/ Association for Computing Machinery; InCoB - International Conference on Bioinformatics; JSBi - Japanese Society for Bioinformatics; PPI - proteinprotein interaction; PTM - post-translational modification; SNP - singlenucleotide polymorphism; TF - transcription factor.

\section{Competing interests}

The authors declare that they have no competing interests.

\section{Authors' contributions}

CS and SR wrote the introduction. CS and SMY (Program Committee Cochairs) managed the review, revision and decision processes. TWT, PH and SR supported the post-acceptance and editorial processing, respectively. All authors have read and approved the final manuscript.

\section{Acknowledgements}

We thank all reviewers for their timely submission of reports. We also thank NOVARTIS Foundation (Japan) for the Promotion of Science, Level Five Ltd. Nabe International Co. Ltd., Amelieff Co. Ltd., and EpiVax Inc. for sponsorships given to GIW/InCoB2015. We are grateful for the material support from AIST Tokyo Waterfront, JSBi and Human Genome Center (University of Tokyo). The excellent support by staff of the APBioNet Secretariat and CBRC was appreciated. Finally, we thank Isobel Peters (BioMed Central) for her support during the prepublication phase. This article has been published as part of BMC Bioinformatics Volume 16 Supplement 18, 2015: Joint 26th Genome Informatics Workshop and 14th International Conference on Bioinformatics: Bioinformatics. The full contents of the supplement are available online at http://www.biomedcentral.com/ bmcbioinformatics/supplements/16/S18.

\section{Authors' details}

'Department of Biology, School of Science and Technology, Nazarbayev University, Astana 010000, Republic of Kazakhstan. ${ }^{2}$ Center for AIDS Research and International Research Center for Medical Sciences, Kumamoto University, Kumamoto 860-0811, Japan. ${ }^{3}$ Computational Biology Research Center, National Institute of Advanced Industrial Science and Technology, Tokyo 135-0064, Japan. ${ }^{4}$ Department of Computational Biology, Graduate School of Frontier Sciences, University of Tokyo, Japan. ${ }^{5}$ Department of Computer Science, Faculty of Engineering, The University of Hong Kong, Hong Kong, HKSAR. ${ }^{6}$ Department of Biochemistry, Yong Loo Lin School of Medicine, National University of Singapore, Singapore 117599. ${ }^{7}$ Department of Chemistry and Biomolecular Sciences, Macquarie University, Sydney NSW 2109, Australia.

\section{Published: 18 December 2015}

\section{References}

1. APBioNet. 2015 [http://www.apbionet.org/]

2. AASBi. 2015 [http:/www.aasbi.org/].

3. GIW/InCoB2015. 2015 [http://incob.apbionet.org/incob15].

4. Bioinformatics. 2015 [http://bioinformatics.oxfordjournals.org/]

5. Joint 26th Genome Informatics Workshop and 14th International Conference on Bioinformatics: Genomics. 2015 [http://www. biomedcentral.com/bmcgenomics/supplements/16/S12].

6. Joint 26th Genome Informatics Workshop and 14th International Conference on Bioinformatics: Bioinformatics. 2015 [http://www. biomedcentral.com/bmcbioinformatics/supplements/16/S18].

7. Joint 26th Genome Informatics Workshop and 14th International Conference on Bioinformatics: Medical Genomics. 2015 [http://www. biomedcentral.com/bmcmedgenomics/supplements/8/S4].
8. Joint 26th Genome Informatics Workshop and 14th International Conference on Bioinformatics: Systems Biology. 2015 [http://www. biomedcentral.com/bmcsystbiol/supplements/9/S6].

9. IEEE/ACM Transactions on Computational Biology and Bioinformatics. 2015 [http://ieeexplore.ieee.org/xpl/Recentlssue.jsp?punumber $=8857$ ].

10. Journal of Bioinformatics and Computational Biology. 2015 [http://www. worldscientific.com/toc/jbcb/13/05].

11. Joint 26th Genome Informatics Workshop and 14th International Conference on Bioinformatics: Genomics. 2015 [http://www. biomedcentral.com/bmcgenomics/supplements/16/S12/11].

12. Wong $Y H$, Wu CC, Lai HY, Jheng BR, Weng HY, Chang TH, et al: Identification of network-based biomarkers of cardioembolic stroke using a systems biology approach with time series data. BMC Syst Biol 2015, 9(Suppl 6):S4.

13. Vandewater $L$, Brusic V, Wilson W, Macaulay L, Zhang P: An adaptive genetic algorithm for selection of blood-based biomarkers for prediction of Alzheimer's disease progression. BMC Bioinformatics 2015, 16(Suppl 18): S1.

14. Wu C, Schwartz JM, Brabant G, Peng SL, Nenadic G: Constructing a molecular interaction network for thyroid cancer via large-scale text mining of gene and pathway events. BMC Syst Biol 2015, 9(Suppl 6):S5.

15. Cheung VG, Spielman RS: Genetics of human gene expression: mapping DNA variants that influence gene expression. Nat Rev Genet 2009, 10:595-604.

16. Picchetti T, Chiquet J, Elati M, Neuvial P, Nicolle R, Birmelé E: A model for gene deregulation detection using expression data. BMC Syst Biol 2015, 9(Suppl 6):S6.

17. Elati M, Neuvial P, Bolotin-Fukuhara M, Barillot E, Radvanyi F, Rouveirol C: LICORN: learning cooperative regulation networks from gene expression data. Bioinformatics 2007, 23(18):2407-2414.

18. Taguchi YH: Identification of aberrant gene expression associated with aberrant promoter methylation in primordial germ cells between E13 and E16 rat F3 generation vinclozolin lineage. BMC Bioinformatics 2015, 16(Suppl 18):S16.

19. Franssens $V$, Bynens $T$, Van den Brande J, Vandermeeren $K$, Verduyckt $M$, Winderickx J: The benefits of humanized yeast models to study Parkinson's disease. Oxid Med Cell Longev 2013, 2013:760629.

20. Wu WS, Wang CC, Jhou MJ, Wang YC: YAGM: a web tool for mining associated genes in yeast based on diverse biological associations. BMC Syst Biol 2015, 9(Suppl 6):S1.

21. Wu WS, Lai FJ: Functional redundancy of transcription factors explains why most binding targets of a transcription factor are not affected when the transcription factor is knocked out. BMC Syst Biol 2015, 9(Suppl 6):S2.

22. Lai FJ, Chang HT, Wu WS: PCTFPeval: a web tool for benchmarking newly developed algorithms for predicting cooperative transcription factor pairs in yeast. BMC Bioinformatics 2015, 16(Suppl 18):S2

23. Stoney RA, Ames RM, Nenadic G, Robertson DL, Schwartz JM: Disentangling the multigenic and pleiotropic nature of molecular function. BMC Syst Biol 2015, 9(Suppl 6):S3.

24. Konishi T: Principal component analysis for designed experiments. BMC Bioinformatics 2015, 16(Suppl 18):S7.

25. Kandala S, Kim IM, Su H: Neddylation and deneddylation in cardiac biology. Am J Cardiovasc Dis 2014, 4(4):140-58

26. Yavuz AS, Sözer NB, Sezerman OU: Prediction of neddylation sites from protein sequences and sequence-derived properties. BMC Bioinformatics 2015, 16(Suppl 18):S9.

27. Kao HJ, Huang $\mathrm{CH}$, Bretaña $N A$, Lu $C T$, Huang $K Y$, Weng $S L$, et al: A twolayered machine learning method to identify protein O-GIcNAcylation sites with O-GICNAc transferase substrate motifs. BMC Bioinformatics 2015, 16(Suppl 18):S10.

28. Sowmya G, Ranganathan S: Discrete structural features among interface residue-level classes. BMC Bioinformatics 2015, 16(Suppl 18):S8,

29. Srinivasulu YS, Wang JR, Hsu KT, Tsai MJ, Charoenkwan P, Huang WL, Huang HL, Ho SY: Characterizing informative sequence descriptors and predicting binding affinities of heterodimeric protein complexes. BMC Bioinformatics 2015, 16(Suppl 18):S14.

30. Ren J, Liu Q, Ellis J, Li J: Positive-unlabeled learning for the prediction of conformational B-cell epitopes. BMC Bioinformatics 2015, 16(Suppl 18):S12.

31. Coltelli P, Barsanti L, Evangelista V, Frassanito AM, Gualtieri P: Water monitoring: automated and real time identification and classification of 
algae using digital microscopy. Environ Sci Process Impacts 2014, 16(11):2656-2665.

32. Kien LL, Li-Lee C, Dhillon SK: Automated identification of copepods using digital image processing and artificial neural network. BMC Bioinformatics 2015, 16(Suppl 18):S4

33. Sun S, Wang X, Gao X, Ren L, Su X, Bu D, et al: Condensing Raman spectrum for single-cell phenotype analysis. BMC Bioinformatics 2015, 16(Suppl 18):S15.

34. Chang CW, Lee WB, Chen-Deng A, Liu T, Tseng JT, Chang DTH: Light-RCV: a lightweight read coverage viewer for next generation sequencing data. BMC Bioinformatics 2015, 16(Suppl 18):S11.

35. Kimura K, Koike A: Analysis of genomic rearrangements by using the Burrows-Wheeler transform of short-read data. BMC Bioinformatics 2015, 16(Suppl 18):S5.

36. Chen $Y L$, Lee $C C$, Lin $Y L$, Yin $K M$, Ho CL, Liu T: Obtaining long $16 S$ rDNA sequences using multiple primers and its application on dioxincontaining samples. BMC Bioinformatics 2015, 16(Suppl 18):S13.

37. Jayasundara D, Saeed I, Chang BC, Tang SL, Halgamuge SK: Accurate reconstruction of viral quasispecies spectra through improved estimation of strain richness. BMC Bioinformatics 2015, 16(Suppl 18):S3.

38. Presidential Commission for the Study of Bioethical Issues. 2012. Privacy and progress in whole genome sequencing. 2015 [http://bioethics.gov/ $\mathrm{cms} /$ sites/default/files/PrivacyProgress508.pdf].

39. Hormozdiari F, Joo JW, Wadia A, Guan F, Ostrosky R, Sahai A, et al: Privacy preserving protocol for detecting genetic relatives using rare variants. Bioinformatics 2014, 30(12):i204-211.

40. He D, Furlotte NA, Hormozdiari F, Joo JW, Wadia A, Ostrovsky R, et al: Identifying genetic relatives without compromising privacy. Genome Res 2014, 24(4):664-672.

41. Shimizu K, Nuida K, Arai H, Mitsunari S, Attrapadung N, Hamada M, et al: Privacy-preserving search for chemical compound databases. BMC Bioinformatics 2015, 16(Suppl 18):S6.

42. International Conference on Bioinformatics 2016 (InCoB2016). 2015 [http://incob16.apbionet.org/].

Cite this article as: Schönbach et al.: GIW and InCoB are advancing bioinformatics in the Asia-Pacific. BMC Bioinformatics 2015 16(Suppl 18):11.

\section{Submit your next manuscript to BioMed Central and take full advantage of:}

- Convenient online submission

- Thorough peer review

- No space constraints or color figure charges

- Immediate publication on acceptance

- Inclusion in PubMed, CAS, Scopus and Google Scholar

- Research which is freely available for redistribution

Submit your manuscript at www.biomedcentral.com/submit
Biomed Central 